\title{
Relationship between Transvaginal Ultrasound Endometrial Thickness, Body Mass Index and Endometrial Pathology in Women with Post-Menopausal Bleeding
}

\author{
Ahmed Farouk Mohamed ${ }^{1, *}$ MB BCh, Ismail Talat El Garhey ${ }^{2}$ MD, Adel Aly Elboghdady ${ }^{2}$ MD
}

\author{
* Corresponding Author: \\ Ahmed Farouk Mohamed \\ ahmedfaroukm91@gmail.com
}

Received for publication April 28 2021; Accepted June 27, 2021; Published online June 27, 2021.

Copyright The Authors published by Al-Azhar University, Faculty of Medicine, Cairo, Egypt. Users have the right to read, download, copy, distribute, print, search, or link to the full texts of articles under the following conditions: Creative Commons Attribution-Share Alike 4.0 International Public License (CC BY-SA 4.0)

doi: $10.21608 /$ aimj.2021.62095.1460

${ }^{I}$ Msc student at Department of Obstetrics \& Gynecology, Al-Azhar University, Cairo, Egypt and Resident at Obstetrics \& Gynecology Department, Shobra General Hospital, Cairo, Egypt.

${ }^{2}$ Department of Obstetrics \& Gynecology, Al-Azhar University, Cairo, Egypt.

\begin{abstract}
Background: Postmenopausal bleeding can be due to benign or malignant pathologies. Body mass index (BMI) is one of the best measures of obesity predicting the risk of endometrial cancer. Postmenopausal bleeding can be assessed using both transvaginal ultrasound and histopathology.

Aim of the work: To Explore the relationship between transvaginal ultrasonography endometrial thickness, Body Mass Index and endometrial pathology in women with postmenopausal bleeding.

Patients and Methods: Our prospective cohort study included 50 patients with postmenopausal bleeding recruited from Al-Hussein University Hospital's gynecology outpatient. All patients had postmenopausal bleeding and exposed to BMI measurements, transvaginal ultrasound assessment of the endometrium and the results correlated to the histopathological presentation of the endometrium.

Results: The age ranged between 46-75years, with the mean 59.16 \pm 5.30 years, the age at menopause ranged between $46-55$ with the mean $49.95 \pm 4.21$, BMI ranged between 25.5-34.7 with the mean $31.57 \pm 1.71$, parity ranged from nullipara to 8 , with the median 4 and the mean endometrial thickness $(\mathrm{mm}) 18.98 \pm 6.95$. According to histopathology, the number of Hyperplasia cases was (29 cases, $58.0 \%$ ), Proliferative (10 cases, 20.0\%), Atrophic (6 cases, 12.0\%) and Malignancy (5 cases, $10.0 \%)$. Patients with endometrial carcinoma had a higher BMI and endometrial thickness than those with benign endometrial pathology.

Conclusion: Obesity is a risk factor in the development of endometrial carcinoma. All overweight patients with postmenopausal bleeding must be properly evaluated to exclude the possibility of malignancy.
\end{abstract}

Keywords: Transvaginal Ultrasound; Body Mass Index; Endometrial Pathology; Post-Menopausal Bleeding.

Disclosure: The authors have no financial interest to declare in relation to the content of this article. The Article Processing Charge was paid for by the authors. Authorship: All authors have a substantial contribution to the article.

\section{INTRODUCTION}

Menopause is described as a series of psychological and physical changes in a woman's life following the cessation of menstruation, and it is derived from the Greek terms "men" and "pause".

Recurrent attacks of bleeding that occur 12 months after the last normal cycle are known as postmenopausal bleeding. ${ }^{2}$

Postmenopausal bleeding may be caused by benign or malignant pathologies. Anatomical changes in the genital tract, infection, endocrinology disorders, malignancies, and systemic disorders are all possible causes. $^{3}$

Vaginal or endometrial atrophy, as well as hormone therapy, are the most common causes of irregular bleeding in postmenopausal women; endometrial cancer accounts for around $10 \%$ of postmenopausal bleeding. ${ }^{4}$
Transvaginal ultrasound can help tailor endometrial biopsy procedures to the person or confidently rule out further examination in women at low risk of endometrial cancer. ${ }^{5}$

Transvaginal ultrasonography revealed endometrium with a low risk of malignancy in women with an endometrial thickness of less than $5 \mathrm{~mm}$, but this risk increased as the endometrial thickness increased, so ultrasound can help us differentiate low-risk patients from high-risk patients. ${ }^{6}$

Endometrial biopsy is the best standard for diagnosing endometrial tissue abnormalities in patients with PMB with high sensitivity ranges. ${ }^{7}$

Age over 40 years is widely considered a risk factor for endometrial disease and an indication for biopsy in women with abnormal bleeding. Endometrial hyperplasia and cancer are more commonly detected in older than in younger women, but the length of 
time exposed to unopposed estrogen stimulation is the most critical risk factor. ${ }^{8}$

The body mass index (BMI) is one of the most accurate indicators of obesity as an endometrial cancer risk factor. ${ }^{9}$

A high BMI has been linked to an increased risk of endometrial cancer. In affluent cultures, obesity is thought to be responsible for around $40 \%$ of endometrial cancer cases. ${ }^{10}$

Our study designed to explore the link between body mass index, TVU endometrial thickness measurement and their relationship to endometrial pathology in postmenopausal bleeding patient.

\section{PATIENTS AND METHODS}

This study was done at Obstetrics \& Gynecology Department, Al-Hussein University Hospital between March to December 2020. Patients were selected from gynecology outpatient. It is a prospective cohort study. Include 50 women with postmenopausal bleeding.

After proper counseling, the ethics committee approved the report. All of the women were approached and written informed consent was given to them.

All cases presented with postmenopausal bleeding and all exposed to measurement of Body Mass Index and transvaginal ultra-sonographic assessment of the endometrium and the results correlated to the histopathological picture of the endometrium.

Criteria for Inclusion: Women that have reached menopause with bleeding after menopause $\geq 12$ months, Endometrial thickness by Transvaginal Ultrasound (TVUS) $\geq 5 \mathrm{~mm}$, Body Mass Index $\geq 25$.

Criteria for Inclusion: women with chronic diseases such as diabetes, hypertension, and hematological disorders, Hormone replacement therapy, Tamoxifen or anticoagulant therapy were given to the women, Presence of an adnexal mass detected by TVUS or apparent causes of cervix or vaginal bleeding, such as ulcers, were removed from the study.

All patients selected for our study were subjected to the following: Full history taking including age, age at the time of menopause, parity, present history of bleeding, history of previous curettage and family history of a related condition.

General and Local examination including inspection of external genetalia, bimanual examination to detect the size of uterus, position, tenderness, mobility, any cervical masses, any adnexal masses and Cusco examination.

Laboratory investigation including (CBC, INR, SGOT, SGPT, SERUM CREATININE, Fasting and postprandial blood sugar).

Transvaginal ultrasound was done using GE LOGIQ V3 Two-dimensional ultrasonography machine equipped with $3.5 \mathrm{MHz}$ transvaginal probe $2 \mathrm{D}$ system at TALAT UNITE El-Hussein university hospital. Endometrium was scanned from cornu to cornu in the sagittal plane of the uterus and endometrial thickness was measured between the two basal layers on the anterior and posterior uterine walls. Calipers positioned perpendicular to the outer edge of the endometrium. We subtracted the fluid diameter if it was used in the double endometrial stripe.

Conservative dilatation and curettage was done with a sharp-ended curette, starting with the fundus, then the posterior wall, anterior wall, right and left lateral walls, and finally the fundus. Until histopathological examination, the specimen is preserved in $10 \%$ formalin.

Statistical Analysis: The data were statistically analyzed using the statistical software for social sciences, version 20.0. (SPSS Inc., Chicago, Illinois, USA). To express quantitative data, the mean and standard deviation were used (SD). To express qualitative data, frequency and percentage were used. An independent-samples t-test of significance was used to compare two means. Mann Whitney is a fictional character who appears in the film Mann Whitney. The U test is used to compare two groups in non-parametric data. The Chi-square (x2) test of significance was used to compare proportions between qualitative parameters. The degree of association between two sets of variables was determined using Pearson's correlation coefficient $(r)$ test. The overall predictivity of the variable and the best cut-off value, as well as the sensitivity and specificity at this cut-off value, were investigated using the receiver operating characteristic (ROC curve) process. P-values of less than 0.05 were considered significant, while P-values of less than 0.001 were considered extremely significant. A Pvalue of more than 0.05 was also considered nonsignificant.

\section{RESULTS}

In terms of age, gravidity, and parity, there was a substantial difference between the two sample groups, as well as a highly significant difference in BMI. There was no noticeable difference in age at menopause, length of PMB, or use of hormonal contraceptives between women with benign and malignant lesions (Table 1).

\begin{tabular}{|c|c|c|c|c|}
\hline Characteristics & Benign & Malignant & Test & p-value \\
\hline Age (years) & $55.29 \pm 5.00$ & $63.03 \pm 5.59$ & $t=3.250$ & $0.002 *$ \\
\hline Gravidity & $4(3-5)$ & $6(2-9)$ & $\mathrm{z}=2.121$ & $0.039 *$ \\
\hline Parity & $3(2-4)$ & $5(1-6)$ & $\begin{array}{l}z=2.019 \\
\end{array}$ & $0.049 *$ \\
\hline $\begin{array}{l}\text { Age at } \\
\text { menopause } \\
\text { (years) }\end{array}$ & $48.41 \pm 3.76$ & $51.4 \mathrm{C}$ & $\mathrm{t}=1.701$ & 0.096 \\
\hline $\begin{array}{l}\text { Duration of } \\
\text { PMB (months) }\end{array}$ & $3.78 \pm 4.31$ & $4.86 \pm 5.59$ & $t=0.517$ & 0.608 \\
\hline BMI $\left(\mathrm{kg} / \mathrm{m}^{2}\right)$ & $28.54 \pm 1.25$ & $34.60 \pm 2.16$ & $t=9.526$ & $<0.001 * *$ \\
\hline $\begin{array}{l}\text { Hormonal } \\
\text { contraception }\end{array}$ & & & & \\
\hline Users & $7(15.6 \%)$ & $2(40.0 \%)$ & \multirow{2}{*}{0.537} & \multirow{2}{*}{0.464} \\
\hline Non users & $38(84.4 \%)$ & $3(60.0 \%)$ & & \\
\hline
\end{tabular}

Table (1): Histopathological comparison of benign and malignant tumors based on demographic data 
The ultra-sonographic findings named adenomyosis, fibroid. There was a substantial difference in endometrial margin between the two sample groups, as well as an extremely significant difference in endometrial thickness. The endometrial echogenicity did not vary significantly. (Table 2 ).

\begin{tabular}{|c|c|c|c|c|}
\hline \multirow{2}{*}{\multicolumn{5}{|c|}{ 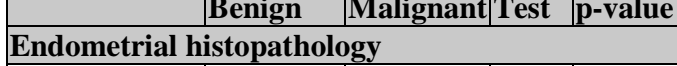 }} \\
\hline & & & & \\
\hline Adenomyosis & $16(35.6 \%)$ & $0(0.0 \%)$ & \multirow{3}{*}{8.654} & \multirow{3}{*}{$0.013 *$} \\
\hline Fibroid & $5(11.1 \%)$ & $3(60.0 \%)$ & & \\
\hline Normal & $24(53.3 \%)$ & $2(40.0 \%)$ & & \\
\hline \multicolumn{5}{|c|}{ Endometrial margin } \\
\hline Regular & $40(88.9 \%)$ & $1(20.0 \%)$ & \multirow{2}{*}{10.178} & \multirow{2}{*}{$0.002 *$} \\
\hline Irregular & $5(11.1 \%)$ & $4(80.0 \%)$ & & \\
\hline \multicolumn{5}{|c|}{ Endometrial echogenicity } \\
\hline Homogeneous & $23(51.1 \%)$ & $0(0.0 \%)$ & \multirow[b]{2}{*}{2.899} & \multirow{2}{*}{0.089} \\
\hline Heterogeneous & $22(48.9 \%$ & $5(100.0 \%)$ & & \\
\hline \multicolumn{5}{|c|}{\begin{tabular}{|l} 
Endometrial Thickness (mm) \\
\end{tabular}} \\
\hline Mean \pm SD & $11.14 \pm 4.73$ & $26.81 \pm 9.16$ & 6.339 & $<0.001 * *$ \\
\hline
\end{tabular}

Table (2): Ultra sonographic findings, endometrial margin, endometrial echogenicity, and endometrial thickness were used to compare benign and malignant histopathology $(\mathrm{mm})$

Histopathological data is demonstrated in (Table 3).

\begin{tabular}{|l|c|c|}
\hline Histopathology & No. & \% \\
\hline - Hyperplasia & $\mathbf{2 9}$ & $\mathbf{5 8 . 0 \%}$ \\
\hline Endometrial atypia & 6 & $12.0 \%$ \\
\hline Endometrial without atypia & 15 & $30.0 \%$ \\
\hline Endometrial polyp & 8 & $16.0 \%$ \\
\hline • Proliferative & $\mathbf{1 0}$ & $\mathbf{2 0 . 0 \%}$ \\
\hline • Atrophic & $\mathbf{6}$ & $\mathbf{1 2 . 0 \%}$ \\
\hline - Malignancy & $\mathbf{5}$ & $\mathbf{1 0 . 0 \%}$ \\
\hline
\end{tabular}

Table (3): Distribution of women with postmenopausal bleeding according to their histopathology $(n=50)$.

There was an extremely significant difference in the prevalence of atypia between the two classes, but no statistically significant difference in the presence of polyps. (Table 4).

\begin{tabular}{|l|c|c|c|c|}
\hline & Benign & Malignant & Test & p-value \\
\hline Endometrial atypia & \multicolumn{3}{|l|}{} \\
\hline Yes & $1(2.2 \%)$ & $5(100.0 \%)$ & \multirow{2}{|l|}{32.008} & $<0.001 * *$ \\
\hline No & $\begin{array}{c}44 \\
(97.8 \%)\end{array}$ & $0(0.0 \%)$ & & \\
\hline Endometrial polyp & \multicolumn{3}{|l|}{} & \\
\hline Yes & $8(17.8 \%)$ & $0(0.0 \%)$ & \multirow{2}{*}{0.149} & 0.699 \\
\hline No & $\begin{array}{c}37 \\
(82.2 \%)\end{array}$ & $5(100.0 \%)$ & & \\
\hline
\end{tabular}

Table (4): Comparison between benign and malignant at histopathology according to endometrial atypia and polyp.

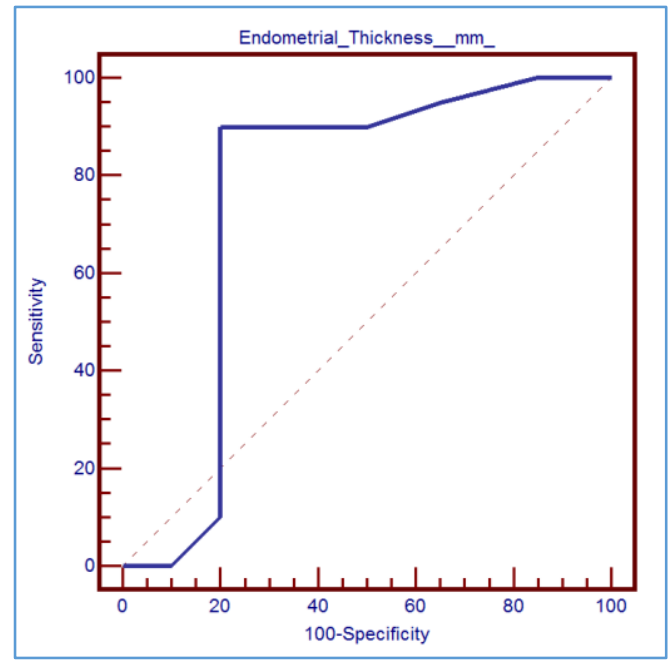

Fig. (1): Receiver-operating characteristic (ROC) curve for prediction of endometrial cancer using the endometrial thickness "mm". Area under the curve (AUC) of 0.859 .

\section{DISCUSSION}

In Postmenopausal Bleeding (PMB) women, malignancy should be considered however atrophic vaginitis or endometritis is the most common cause of postmenopausal bleeding. Women who are experiencing postmenopausal bleeding should be thoroughly evaluated to rule out endometrial cancer, which occurs in just $10 \%$ of cases. ${ }^{11}$

The use of transvaginal ultrasound (TVUS) as a firstline investigation to screen patients with a higher risk of endometrial cancer is common. It is a quite noninvasive procedure with good negative predictive value if properly done 29.Evaluation of postmenopausal bleeding women also includes biopsy of the endometrium. ${ }^{12}$

The age of the postmenopausal women was ranged from 46 to 75 years with the mean of $59.16 \pm 5.30$ years, parity ranged from nullipara to para 8 with a median of para 4 and BMI ranged from 25.5 to 34.7 $\mathrm{kg} / \mathrm{m}^{2}$ with a mean of $31.57 \pm 1.71 \mathrm{~kg} / \mathrm{m}^{2}$.

According to the characteristics of the women, there was significant difference was found between the two study groups concerning age, gravidity and parity and a highly significant difference in regards to BMI.

In the present study, endometrial thickness and BMI were found to have a significant positive relationship.

Khalaf et al. ${ }^{13}$ agreed with us and reported that the malignant group had a statistically highly significant difference in age and BMI compared to the benign group, but disagreed with us on parity, stating that there was a statistically highly significant difference in parity between the two studied groups, with the benign group having more.

Zhang et al. ${ }^{14}$ reported that endometrial cancer risk was 32 percent higher in overweight (BMI 25-30) women and 2.5 times higher in obese (BMI > 30) 
women as compared to women of normal weight (BMI 25). But Folsom et al. ${ }^{15}$ disagreed when adjusted for body mass index, there was no connection between abdominal body fat and the risk of endometrial carcinoma, according to the study.

Also Van den Bosch et al. ${ }^{16}$ after adjusting for age, they found no connection between endometrial thickness and weight or BMI, concluding that age is a major confounder in regression analysis.

Also Schramm et al. ${ }^{17}$ found that People with EC were found to be significantly older than women without cancer, according to our findings. They found no major variations in BMI between women who had EC and those who did not.

Opolskiene et al. ${ }^{18}$ stated that patients with malignant endometrium were found to be older (median age, 73 years vs. 63 years), have a higher BMI (median, 31 $\mathrm{kg} / \mathrm{m} 2$ vs. $27 \mathrm{~kg} / \mathrm{m} 2$ ), and have thicker endometrium (median, $16.2 \mathrm{~mm}$ vs. $9.6 \mathrm{~mm}$ ) than those with benign endometrium.

Friedenreich et al. ${ }^{19}$ found a strong correlation between endometrial cancer risk, obesity, and body fat distribution after analyzing 223000 postmenopausal bleeding women. Obese women (BMI 30 to 40) had a risk ratio of 1.78 as compared to average-weight women, and morbidly obese women (BMI 40) had a risk ratio of 3.02.

And agreed with Zaki et al. ${ }^{20}$ in their study, they discovered that (high BMI) was one of the best markers of obesity for predicting the risk of endometrial cancer in Egyptian women with PMB.

Our findings matched those of Lukanova et al. ${ }^{21}$ who found that obese women (BMI 30) had a 36\% higher cancer risk than women with a BMI in the average range (18.5-25). The endometrium was the cancer site most closely linked to obesity (95 percent confidence interval 1.86-7.43).

Our findings corroborated those of Fourberg and Thune $^{22}$ who discovered 130 cases of endometrial cancer in obese women (BMI 30) with a risk ratio of 2.57 when compared to average-weight women (BMI 25). They came to the conclusion that high energy intake (5044-6401 kJ/day) is linked to a higher risk of cancer in women under 50 years old when compared to low energy intake (4266 kJ/day) (RR: 3.40), as well as the fact that outdoor activities can be beneficial. The RR for non-sedentary obese women drops to 0.18 , implying that, regardless of BMI, inactivity and a high-energy diet are the primary risk factors for endometrial cancer.

This study also agreed with the International Agency for Research on Cancer in classifying the evidence for the connection between obesity and endometrial cancer as 'strong or compelling' and the RR (relative risk) of obese women (BMI 30) compared to nonobese women was found to be two to three times higher. $^{23}$

Our study agreed with Andolf et al. ${ }^{24}$ who discovered a connection between endometrial thickness and BMI. However, they did not include the patients' age or the time after menopause in their study. Tsuda et al. $^{25}$ discovered that there is substantial consensus in postmenopausal women about the relationship between BMI and sonographic endometrial thickness. Several studies have shown that patients with a thin endometrium have a lower risk of endometrial cancer, but different threshold points for a minimum thickness have been proposed.

The mean endometrial thickness in patients with hyperplasia or carcinoma has always been higher than in patients without endometrial disease in all tests.

The majority of studies suggest endometrial thickness values of 4 or $5 \mathrm{~mm}$ as the lower limit for cancer risk, values in this range have a high sensitivity.

With regard to the ultra-sonographic results in the present study, the inclusion criteria of the examined women included that endometrial thickness was $\geq$ $5 \mathrm{~mm}$; as most studies agree with that value to exclude endometrial pathology.

The benign group's mean endometrial thickness was $11.14 \pm 4.73 \mathrm{~mm}$, while the malignant group's was $26.81 \pm 9.16 \mathrm{~mm}$, suggesting a highly statistically significant discrepancy between the two groups since the endometrium was substantially thicker in the malignant group. With a cutoff value of $90 \%$ endometrial thickness and precision of $80 \%$, malignant pathology was calculated to be greater than $17 \mathrm{~mm}$

Our study showed similar results as Karlsson et al. ${ }^{26}$ in the Nordic multi centric study enrolled 1168 patients who were subjected to transvaginal sonography to measure endometrial thickness and then compared endometrial thickness to endometrial histopathology in a prospective randomized controlled trial.

Atrophic endometrium (mean ET 2.4-5.3 mm), carcinoma (mean ET 9-32 mm), and endometrial carcinoma were the three types of findings (mean thickness $21.1 \mathrm{~mm}$ ).

In a survey of 287 patients with PMB, Karasu et al. ${ }^{27}$ discovered that a TVUS determined cut-off value of $4 \mathrm{~mm}$ endometrial thickness has a 99 percent negative predictive value for endometrial cancer. Endometrial sampling is recommended with a cut-off of $4 \mathrm{~mm}$, and 27 of the 287 patients who had endometrial sampling found EC.

This study agrees with Gupta et al. ${ }^{28}$ who found that an endometrial thickness of $5 \mathrm{~mm}$ used as a predictor of endometrial pathology has a sensitivity of 83 percent, a specificity of 77 percent, and a positive predictive value of 54 percent when compared to uterine curettage.

Furthermore, Bruchim et al. ${ }^{29}$ found that the mean endometrial thickness for all patients without cancer was $6.3 \pm 4.3 \mathrm{~mm}$, which was substantially lower than the endometrial thickness of patients with endometrial carcinoma, which was more than 10 $\mathrm{mm}$, and no cases of endometrial carcinoma were 
found in any patients with endometrial thickness less than $5 \mathrm{~mm}$.

However, according to Phillip et al. ${ }^{30}$ the association between imaging and pathology is unreliable. The endometrial thickness in half of their cases of endometrial cancer was between 3 and $4 \mathrm{~mm}$. A benign pathology was found in $70 \%$ of women with endometrial thickness greater than $5 \mathrm{~mm}$.

Furthermore, Eitan et al. ${ }^{31}$ Endometrial thickness as measured by ultrasound ranged from 5 to $32 \mathrm{~mm}$ (mean of $12 \mathrm{~mm}$ ) in 29 patients with endometrial cancer, with all patients diagnosed with cancer and most patients diagnosed with endometrial hyperplasia dropping within a $10 \mathrm{~mm}$ cutoff point.

Furthermore, Salman et al. ${ }^{32}$ In a study of 142 women with $\mathrm{PMB}$, researchers discovered that patients with malignant endometrium were older (53.8 years vs 65.3 years, $\mathrm{P} 0.001$ ) and had a higher mean BMI (27.9 kg/m2 vs $30.6 \mathrm{~kg} / \mathrm{m} 2, \mathrm{P}=0.028)$. Women with endometrial cancer had a marginally longer menopause, and endometrial thickness on TVUS was $8.8 \pm 2.2 \mathrm{~mm}$ in those without cancer versus $26.0 \pm 6.0 \mathrm{~mm}$ in those with cancer.

Schramm et al $^{33}$ found that the thickness of the endometrium was substantially higher in women with EC than in women without, with a median endometrial thickness of $14.3 \mathrm{~mm}$ for patients with EC and $9.0 \mathrm{~mm}$ for patients with no malignancy in 254 women reporting of postmenopausal bleeding for the prediction of endometrial cancer. They observed that none of the study's cutoff points offered ideal diagnostic results when it came to combining the clinically needed high sensitivity with reasonable specificity rates.

In 205 women with postmenopausal bleeding, Granberg et al. ${ }^{34}$ used TVUS to measure endometrial thickness as an indicator of endometrial abnormality, and revealed that if the endometrium was less than 9 $\mathrm{mm}$ deep, no endometrial cancer was found during curettage. The median endometrial thickness in women with endometrial cancer was $18.2 \pm 6.2 \mathrm{~mm}$, compared to $3.4 \pm 1.2 \mathrm{~mm}$ in women with atrophic endometriosis. The positive predictive value for detecting endometrial abnormality was 87.3 percent if the cutoff point for endometrial abnormality was 5 $\mathrm{mm} .70 \%$ of curettage procedures could have been prevented if this cap had been used in this study.

Moodley and Roberts ${ }^{35}$ determined that endometrial thickness of at least $4 \mathrm{~mm}$ in postmenopausal women should be used as a cutoff point to rule out malignancy unless other risk factors exist. In addition, Chandavarkar et al. ${ }^{36}$ found that in a woman with postmenopausal bleeding and an endometrial echo complex (EEC) of less than $4 \mathrm{~mm}$, no further diagnostic procedures should be performed. However, an EEC of less than $4 \mathrm{~mm}$ does not rule out endometrial cancer and does not exclude histological examination.

The median endometrial thickness of women diagnosed with endometrial cancer was $15.7 \mathrm{~mm}$ compared to $3.2 \mathrm{~mm}$ in those who were not diagnosed with cancer, according to Wong et al. ${ }^{37}$ with the variance being statistically important and the
In a meta-analysis of 35 studies comparing the effectiveness of TVS in detecting endometrial abnormalities in postmenopausal bleeding, SmithBindman et al. ${ }^{38}$ recruited 5298 women, 13 percent of whom had uterine cancer as the underlying pathology. The majority of the women had endometrial polyps, while the remainder had benign causes. In our sample, $10 \%$ of women had endometrial carcinoma.

Gredmark et al. $^{39}$ reported that endometrial histopathology was related to the incidence of bleeding in 457 postmenopausal women observed for 18 months in a health care county in Sweden, with 50 percent atrophic endometrium, 10 percent endometrial hyperplasia, 9 percent polyp, 8 percent adenocarcinoma, 4 percent proliferative endometrium, 1 percent secretory endometrium, 14 percent unremarkable endometrium, and 1 percent secret

With 12 percent atrophic endometrium, 10\% carcinoma, 58 percent endometrial hyperplasia, and $20 \%$ proliferative endometrium, our findings are close to those of the previous report. Women with postmenopausal bleeding were treated with dilation and curettage without the use of transvaginal sonography to assess endometrial thickness prior to endometrial sampling in our study, which differs from the previous one.

Our study showed that there was statistically important difference between benign and malignant groups as the ultrasound endometrial margin was more regular in the benign group while it was more irregular in the malignant group and endometrium was heterogeneous in only $48.9 \%$ in the benign group, but all the malignant group had heterogeneous endometrium.

This study agreed with Epstein and Valentin ${ }^{5}$ in that irregular surface and heterogeneous structure of the endometrium were found to be a sign of endometrial malignancy.

With regard to the endometrial pathology in the present study, the most common endometrial pathology was 15 cases $(30.0 \%)$ with endometrial hyperplasia without atypia followed by 10 cases (20.0\%) with disordered proliferative endometrium, 8 cases (16\%) hyperplastic endometrial polyp, 6 cases $(12.0 \%)$ with atrophic endometrium, 6 cases $(12.0 \%)$ with hyperplasia with atypia and 5 cases (10.0\%) with endometrial carcinoma. The presence of endometrial hyperplasia as the most common pathology was explained as most of the studied patients were obese.

These findings are different in comparison to the following studies mostly due to different sample size and inclusion criteria as:

Yaman et al. ${ }^{40}$ studied 213 cases of postmenopausal bleeding they found that 42 cases $(19.7 \%)$ were malignant, 109 cases $(51.17 \%)$ were benign lesions 
(polyp-hyperplasia), and 62 cases $(29.1 \%)$ were atrophic.

Opolskiene et al. ${ }^{18}$ studied 261 cases of postmenopausal bleeding they found that 63 cases (24\%) were malignant, 198 cases $(76 \%)$ were benign lesions.

In terms of the occurrence of endometrial polyp in endometrial pathology, this study discovered that there was no substantial difference between the benign and malignant groups, as it was present in just $17.8 \%$ of the benign group, 82.2 percent was absent, and it was absent in all of the malignant group.

In a systematic review and meta-analysis of the oncogenic potential of endometrial polyps, Lee et $\mathrm{al}^{41}$ in women with endometrial polyps, symptomatic vaginal bleeding and postmenopausal status was related to an increased risk of endometrial malignancy, contrary to our findings.

Endometrial cancer was more common in women with endometrial polyps and postmenopausal bleeding according to Costa-Paiva et al. ${ }^{42}$

\section{CONCLUSION}

Endometrial carcinoma is associated with obesity, which is a major risk factor. All overweight patients with postmenopausal bleeding should be carefully screened to rule out the possibility of cancer.

\section{REFERENCES}

1. Samarasiri DG, Rajanayake IT, Wattegedara PK, et al. Women's Experiences on Menopause: A Phenomenological Study.2017; 12(2):43-62.

2. Meeta M, Digumarti L, Agarwal N, et al. Clinical practice guidelines on menopause: An executive summary and recommendations: Indian menopause society 2019-2020. Journal of Mid-life Health. 2020; 11(2):55.

3. Goodman A. Postmenopausal uterine bleeding. UpToDate. Accessed online June. 2014; 16(3):54-5

4. Neelgund $S$ and Hiremath PB. Abnormal uterine bleeding in perimenopause. Journal of Evolution of Medical and Dental Sciences. 2016; 5(51):3337-42.

5. Epstein E and Valentin L. Gray scale ultrasound morphology in the presence or absence of intrauterine fluid and vascularity as assessed by color Doppler for discrimination between benign and malignant endometrium in women with postmenopausal bleeding. Ultrasound in Obstetrics and Gynecology: The Official Journal of the International Society of Ultrasound in Obstetrics and Gynecology. 2006;28(1), pp.89-95.

6. Ghoubara A, Emovon E, Sundar S, et al. Thickened endometrium in asymptomatic postmenopausal women-determining an optimum threshold for prediction of atypical hyperplasia and cancer. Journal of Obstetrics and Gynaecology. 2018; 38(8):1146-9.

7. Günakan E, Atak Z, Albayrak M, et al. Endometrial histopathology results and evaluation of endometrial cancer risk in geriatric women. Przeglad menopauzalny = Menopause review. 2018; 17(1):18.

8. Fritz MA and Speroff L. Clinical gynecologic endocrinology and infertility. lippincott Williams \& wilkins; 2012.

9. Laskey RA, McCarroll ML and Von Gruenigen VE. Obesity related endometrial cancer: an update on survivorship approaches to reducing cardiovascular death. BJOG: An International Journal of Obstetrics \& Gynaecology. 2016; 123(2):293-8.

10. Constantine GD, Kessler G, Graham S et al. Increased incidence of endometrial cancer following the Women's Health Initiative: an assessment of risk factors. Journal of Women's Health. 2019; 28(2):237-43.

11. Brand AH. The woman with postmenopausal bleeding. Australian family physician. 2007; 36(3):116.

12. Dimitraki M, Tsikouras $\mathrm{P}$, Bouchlariotou $\mathrm{S}$, et al. Clinical evaluation of women with PMB. Is it always necessary an endometrial biopsy to be performed? A review of the literature. Archives of gynecology and obstetrics. 2011; 283(2):261-6.

13. Khalaf MS, Zaki MS, Elshourbgy MS, et al. Relationship between Estradiol Level, Transvaginal Ultrasound Endometrial Thickness, Body Mass Index and Endometrial Pathology in Women with Postmenopausal Bleeding. The Egyptian Journal of Hospital Medicine. 2019; 74(2):284-93.

14. Zhang Y, Liu H, Yang S, et al. Overweight, obesity and endometrial cancer risk: results from a systematic review and meta-analysis. The International journal of biological markers. 2014; 29(1):e21-9.

15. Folsom AR, Kaye SA, Potter JD, et al. Association of incident carcinoma of the endometrium with body weight and fat distribution in older women: early findings of the Iowa Women's Health Study. Cancer research. 1989; 49(23):6828-31.

16. Van den Bosch T, Ameye L, Van Schoubroeck $\mathrm{D}$, et al. Intra-cavitary uterine pathology in women with abnormal uterine bleeding: a prospective study of 1220 women. Facts, views \& vision in ObGyn. 2015; 7(1):17.

17. Schramm A, Ebner F, Bauer E, et al. Value of endometrial thickness assessed by transvaginal ultrasound for the prediction of endometrial cancer in patients with postmenopausal bleeding. Archives of gynecology and obstetrics. 2017; 296(2):319-26. 
18. Opolskiene G, Sladkevicius P, Jokubkiene L, et al. Three dimensional ultrasound imaging for discrimination between benign and malignant endometrium in women with postmenopausal bleeding and sonographic endometrial thickness of at least $4.5 \mathrm{~mm}$. Ultrasound in Obstetrics and Gynecology: The Official Journal of the International Society of Ultrasound in Obstetrics and Gynecology. 2010; 35(1):94-102.

19. Friedenreich C, Cust A, Lahmann PH, et al. Anthropometric factors and risk of endometrial cancer: the European prospective investigation into cancer and nutrition. Cancer Causes \& Control. 2007; 18(4):399-413.

20. Zaki A, Gaber A, Ghanem E, et al. Abdominal obesity and endometrial cancer in Egyptian females with postmenopausal bleeding. Nutrition and cancer. 2011; 63(8):1272-8.

21. Lukanova A, Björ O, Kaaks R, et al. Body mass index and cancer: results from the Northern Sweden Health and Disease Cohort. International journal of cancer. 2006; 118(2):458-66.

22. Furberg AS and Thune I. Metabolic abnormalities (hypertension, hyperglycemia and overweight), lifestyle (high energy intake and physical inactivity) and endometrial cancer risk in a Norwegian cohort. International journal of cancer. 2003; 104(6):669-76.

23. IARC Working Group on the Evaluation of Carcinogenic Risks to Humans, International Agency for Research on Cancer. Some traditional herbal medicines, some mycotoxins, naphthalene and styrene. World Health Organization; 2002.

24. Andolf EL, Dahlander K and Aspenberg P. Ultrasonic thickness of the endometrium correlated to body weight in asymptomatic postmenopausal women. Obstetrics and gynecology. 1993; 82(6):936-40.

25. Tsuda H, Kawabata M, Kawabata $\mathrm{K}$ et al. Improvement of diagnostic accuracy of transvaginal ultrasound for identification of endometrial malignancies by using cutoff level of endometrial thickness based on length of time since menopause. Gynecologic oncology. 1997; 64(1):35-7.

26. Karlsson B, Granberg S, Wikland M, et al. Transvaginal ultrasonography of the endometrium in women with postmenopausal bleeding - a Nordic multicenter study. American journal of obstetrics and gynecology. 1995; 172(5):1488-94.

27. Karasu AF, Ates S, Gurbuz T, et al. A Clinicopathological Study of Transvaginal Endometrial Thickness Measurement in Asymptomatic Postmenopausal Patients and Patients with Postmenopausal Bleeding. Gynecology Obstetrics \& Reproductive Medicine. 2019; 25(2):85-8.
28. Gupta JK, Wilson S and Desai P. How should we investigate women with postmenopausal bleeding? Acta obstetricia et gynecologica Scandinavica. 1996; 75(5):475-9.

29. Bruchim I, Biron Shental T, Altaras MM, et al. Combination of endometrial thickness and time since menopause in predicting endometrial cancer in women with postmenopausal bleeding. Journal of Clinical Ultrasound. 2004; 32(5):219-24.

30. Phillip H, Dacosta V, Fletcher $H$, et al. Correlation between transvaginal ultrasound measured endometrial thickness and histopathological findings in Afro-Caribbean Jamaican women with postmenopausal bleeding. Journal of Obstetrics and Gynecology. 2004; 24(5):568-72.

31. Eitan R, Saenz CC, Venkatraman ES, et al. Pilot study prospectively evaluating the use of the measurement of preoperative sonographic endometrial thickness in postmenopausal patients with endometrial cancer. Menopause. 2005; 12(1):27-30.

32. Salman MC, Bozdag G, Dogan S, et al. Role of postmenopausal bleeding pattern and women's age in the prediction of endometrial cancer. Australian and New Zealand Journal of Obstetrics and Gynecology. 2013; 53(5):484-8.

33. Schramm A, Ebner F, Bauer E, et al. Value of endometrial thickness assessed by transvaginal ultrasound for the prediction of endometrial cancer in patients with postmenopausal bleeding. Archives of gynecology and obstetrics. 2017; 296(2):319-26.

34. Granberg S, Wikland M, Karlsson B, et al. Endometrial thickness as measured by endovaginal ultrasonography for identifying endometrial abnormality. American journal of obstetrics and gynecology. 1991; 164(1):47-52.

35. Moodley M and Roberts C. Clinical pathway for the evaluation of postmenopausal bleeding with an emphasis on endometrial cancer detection. Journal of Obstetrics and Gynecology. 2004; 24(7):736-41.

36. Chandavarkar U, Kuperman JM, Muderspach LI, et al. Endometrial echo complex thickness in postmenopausal endometrial cancer. Gynecologic oncology. 2013; 131(1):109-12.

37. Wong AW, Lao TH, Cheung $\mathrm{CW}$, et al. Reappraisal of endometrial thickness for the detection of endometrial cancer in postmenopausal bleeding: a retrospective cohort study. BJOG: An International Journal of Obstetrics \& Gynecology. 2016; 123(3):439-

38. Smith Bindman R, Weiss E and Feldstein V. How thick is too thick? When endometrial thickness should prompt biopsy in postmenopausal women without vaginal bleeding. Ultrasound in Obstetrics and Gynecology: The Official Journal of the International Society of Ultrasound in 
Obstetrics and Gynecology. 2004; 24(5):55865.

39. Gredmark T, Kvint S, Havel $G$, et al. Histopathological findings in women with postmenopausal bleeding. BJOG: An International Journal of Obstetrics \& Gynaecology. 1995; 102(2):133-6.

40. Yaman C, Habelsberger A, Tews G, et al. The role of three-dimensional volume measurement in diagnosing endometrial cancer in patients with postmenopausal bleeding. Gynecologic oncology. 2008; 110(3):390-5.
41. Lee SC, Kaunitz AM, Sanchez-Ramos L, et al. The oncogenic potential of endometrial polyps: a systematic review and meta-analysis. Obstetrics \& Gynecology. 2010; 116(5):1197205.

42. Costa-Paiva L, Godoy Jr CE, Antunes Jr A, et al. Risk of malignancy in endometrial polyps in premenopausal and postmenopausal women according to clinicopathologic characteristics. Menopause. 2011; 18(12):1278-82. 